\title{
Research progress on GP73 in malignant tumors
}

This article was published in the following Dove Press journal:

OncoTargets and Therapy

\section{Rong Liang \\ Ziyu Liu \\ Xuemin Piao \\ Mingtang Zuo \\ Jinyan Zhang \\ Zhihui Liu \\ Yongqiang Li \\ Yan Lin}

Department of Medical Oncology, Affiliated Tumor Hospital of Guangxi Medical University, Nanning, Guangxi 53002 I, People's Republic of China

Correspondence: Yan Lin Department of Medical Oncology, Affiliated Tumor Hospital of Guangxi Medical University, 7I Hedi Road, Nanning, Guangxi 53002I, People's Republic of China

Tel/fax +86 77I 5335155

Email linyanmgx@I63.com

\begin{abstract}
Malignant cancer is one of the most serious diseases that currently endanger human health. As most tumors are diagnosed at an advanced stage, the current treatments show poor therapeutic efficacy, and the patients have poor prognosis. However, a 5-year survival rate higher than $80 \%$ could be achieved if tumors are diagnosed at an early stage. Therefore, early diagnosis and treatment play important roles in the prevention and treatment of malignant tumors, and serum tumor markers are important for the early diagnosis of malignant cancers. Recent studies have shown that GP73, a transmembrane protein, has greater diagnostic value in primary liver cancer than in other types of cancers, and research on the regulation of GP73 expression has unveiled broad prospects in anticancer targeted therapy. Thus, GP73, as a new tumor marker, deserves further study.
\end{abstract}

Keywords: GP73, malignant tumors, tumor marker, diagnosis, prognosis

\section{Introduction}

Cancer has become the primary cause of human death and the largest public health problem worldwide, and the morbidity and mortality rates associated with cancer continue to increase. ${ }^{1}$ The clinical importance of tumor markers in the early treatment of cancer is becoming increasingly recognized. Golgi glycoprotein 73 (Golgi protein-73, GP73; also known as Golgi membrane protein 1 [GOLM1] or Golgi membrane protein [GOLPH2]) is a transmembrane glycoprotein that was first discovered in $2000 .^{2}$ The current literature suggests that GP73 is one of the most valuable markers of liver cancer and participates in the development of liver cancer through multiple pathways. It was recently reported that GP73 is highly expressed in gallbladder cancer (GBC), lung cancer, prostate cancer $(\mathrm{PCa})$, and other cancers and is associated with prognosis, which indicates that GP73 has a potential clinical value in the diagnosis of multiple tumors. Therefore, this study reviews the recent studies on GP73 and malignant tumors.

\section{Identification and characterization of GP73}

GP73 is also called type II GOLPH2 and GOLM1. Its coding gene is located at chromosome 9p21.33, and its full-length sequence is 3,080 bp, comprising a 1,200-bp open reading frame harboring two regions coding products composed of 400 and 391 amino acids. It was first discovered in 2000 by the American scholar Kladney et $\mathrm{al},{ }^{2}$ who studied adult giant cell hepatitis $(\mathrm{GCH})$. In normal cells, GP73 resides in the cis and medial-Golgi cisternae and consists of three domains: the cytoplasmic, transmembrane, and Golgi lumen domains. The $\mathrm{N}$ terminus is hydrophobic, the single transmembrane region contains a signal peptide cleavage site, and the $\mathrm{C}$ terminus has five glycosylation sites, one alpha helical domain, and one acid tail and serves as a functional protein-protein interaction domain. Some diseases could induce the release of GP73 in the cis and medial-Golgi cisternae, and the released molecules could then 
be transported through endosomes until being secreted into the circulation. Kladney et al used the Northern blot method to examine GP73 mRNA expression in 50 human tissues and found that high expression levels are concentrated in epithelial cells, whereas GP73 mRNA is rarely expressed in muscle, lymphoid tissue, or white blood cells.

\section{GP73 and hepatocellular carcinoma (HCC)}

$\mathrm{HCC}$ is one of the most common malignant tumors in the world. Alpha-fetoprotein (AFP) is a world-recognized serum marker for the diagnosis of liver cancer, and many recent studies have suggested that GP73 has greater sensitivity and specificity than AFP. In fact, the sensitivity of GP73 generally ranges from $69 \%$ to $83 \%$, and its specificity ranges from $73 \%$ to $97 \%{ }^{3}$ Dynamic changes in the GP73 levels reflect the effects of surgery and transcatheter arterial chemoembolization (TACE) and can be used as a reference indicator of prognosis and disease progression. ${ }^{4,5}$ A previous study also found that GP73 mRNA is associated with tumor size, vascular infiltration, and tumor differentiation, which suggests that it can be used as an indicator of tumor progression. ${ }^{6}$ However, the use of GP73 in the early diagnosis of HCC has some challenges. Liu et $\mathrm{al}^{7}$ concluded that the presence of autogp73 antibodies in serum would interfere with the monitoring of serum GP73, and the results obtained for some patients with liver cirrhosis and some with HCC were not significantly different. Therefore, it is necessary to further study and explore the mechanism of action of GP73 to assess whether it is a suitable early diagnostic index for HCC.

GP73 plays an important role in the maintenance of normal liver function, and scientists have explored its functions and downstream signal transduction mechanisms in HCC. ${ }^{8}$ Ye et $\mathrm{al}^{9}$ found that GP73 selectively interacts with the EGFR and thereby helps EGFR and other receptor tyrosine kinases (RTKs) anchor across the trans-Golgi network (TGN) and recycle cell membranes to promote HCC metastasis; thus, GP73 is considered a key promoter of HCC metastasis. A study conducted by Jin et $\mathrm{al}^{10}$ showed that GP73 enhances the expression of matrix metalloproteinase-13 (MMP-13) through transcriptional activation mediated by cAMP response elementbinding protein (CREB) and that the GP73-CREB-MMP-13 axis enhances the invasive ability of cancer cells. The abnormal expression of miRNAs plays an important role in the development of HCC, and a study by Zhang et $\mathrm{al}^{11}$ indicated that GP73 is a direct target of miR-382. The knockdown of GP73 might inhibit the miR-382-mediated migration and invasion of $\mathrm{HCC}$ cells. Zhao et al ${ }^{12}$ found that GP73 is also regulated by miR493-5p and that the levels of these two factors are negatively correlated. HCC tissues show significant downregulation of miR-493-5p and abnormal upregulation of GP73, which promotes HCC proliferation. The epithelial-mesenchymal transition (EMT) is closely related to the invasion and metastasis of cancer cells. A previous study found marked changes in the phenotypic characteristics of the EMT and related protein expression after GP73 gene silencing. Specifically, silencing of the GP73 gene reduced the expression of the key EMT factors $\mathrm{N}$-cadherin and E-cadherin, thereby decreasing cell adhesion and promoting cancer cell movement. ${ }^{13,14}$ Zhou et $\mathrm{al}^{15}$ found that in addition to inducing autophagy, GP73 might also be involved in the autophagy degradation process. These previous studies indicate that GP73 is involved in the development of HCC through multiple mechanisms.

Preliminary studies have explored the effect of GP73 in the treatment of HCC. Wang et $\mathrm{al}^{16}$ first designed the adenovirus GD55 by replacing the endogenous E1A promoter with the GP73 promoter. The results showed that GD55 led to decreased tumor proliferation and had fewer side effects on normal liver cells. Oxaliplatin (OXA) is a third-generation platinum-based drug that is currently considered a relatively effective chemotherapeutic for $\mathrm{HCC}$ patients. Ye et al ${ }^{17}$ induced drug resistance by increasing the drug concentration and found that the knockdown of GP73 in the $97 \mathrm{H}$ cell line resulted in a significantly lower IC50 of OXA and significantly reduced cell proliferation. GP73 is expressed in the OXA-resistant Hep3B cell line, and the resulting change in the $\mathrm{IC}_{50}$ of OXA led to increased cell proliferation capacity. This finding indicates that GP73 plays an important role in OXA resistance in $\mathrm{HCC}$ cell lines.

\section{GP73 and GBC}

GBC is a common malignant tumor in the biliary system and is the sixth most common cancer of the digestive system. ${ }^{1}$ Because the mortality rate associated with GBC is high and these tumors have few distinguishing symptoms, the early diagnosis of GBC is crucial. Liu et $\mathrm{al}^{18}$ found that the GP73 expression levels show gradual increases from the normal gallbladder to gallbladder adenomatous polyps and GBC, which suggests that GP73 might be a tumor marker of GBC. A statistical analysis of clinical features found that positive GP73 expression is related to the degree of GBC differentiation, Nevin stage, and lymph node metastasis. The rate of GP73 positivity in GBC with an advanced Nevin stage and lymph node metastasis was higher than that in non-metastatic GBC. This finding suggests that GP73 might be involved in GBC invasion and lymph node metastasis and likely plays an important role in the biological behavior of GBC. 


\section{GP73 and lung cancer}

Lung cancer is one of the most harmful malignant tumors to humans worldwide. The vast majority of lung cancer cases in clinical practice are non-small-cell lung cancer (NSCLC), and $70 \%$ of the patients with lung cancer are diagnosed with late-stage disease. Many scientists believe that further study on GP73 could result in its development as a marker for the early diagnosis of NSCLC. ${ }^{19}$ Han et $\mathrm{al}^{20}$ collected 90 primary NSCLC tumor tissue samples and 19 distal matched normal lung tissue samples and found positive GP73 expression in the NSCLC group, with a significantly higher positivity rate $(56 \%)$ than that in the normal lung group. Zhang et $\mathrm{al}^{21}$ and Aruna and $\mathrm{Li}^{22}$ obtained the same results: GP73 expression in lung adenocarcinoma tissue is higher than that in lung squamous cell carcinoma tissue, and GP73 is only slightly expressed in mesenchymal cells and normal tissues adjacent to cancer. These findings suggest that GP73 could be a potential diagnostic indicator of NSCLC.

Similar to the results obtained in the studies of GP73 in $\mathrm{HCC}$, the results obtained by Aruna and $\mathrm{Li}^{22}$ revealed that the overexpression of GP73 significantly promotes the growth of NSCLC cells in vivo, whereas the inhibition of endogenous GP73 has the opposite result. High GP73 expression can induce the EMT, thus promoting the proliferation, migration, and invasion of NSCLC cells. In addition, these researchers confirmed that GP73 enhances the invasiveness of NSCLC by activating MMP-13 signaling. In conclusion, their results suggest that GP73 overexpression promotes the progression of NSCLC and that GP73 might be a new therapeutic target for the treatment of this disease.

\section{GP73 and PCa}

$\mathrm{PCa}$ is the fourth most common malignant tumor in males and one of the leading causes of cancer-related death in males, and its incidence increases with age. ${ }^{1} \mathrm{Li}$ and Zhong ${ }^{23}$ and Kristiansen et $\mathrm{al}^{24}$ confirmed that GP73 mRNA and protein are upregulated in $\mathrm{PCa}$ and that GP73 expression is significantly higher in androgen-independent $\mathrm{PCa}$ cells than in androgen-dependent PCa cells. In addition, the GP73 expression levels are higher in $\mathrm{PCa}$ than in benign prostatic hyperplasia and normal prostatic tissue. Experiments conducted by Kristiansen et $\mathrm{al}^{24}$ revealed that screening for GP73 in urine can detect PCa with higher sensitivity than the traditional prostate-specific antigen (PSA) blood test. Laxman et $\mathrm{al}^{25}$ reached the same conclusion and found that the GP73 positivity rate is also high among alpha-methyl acyl-CoA racemase (AMACR)-negative PCa cases. These data suggest that GOLM1 is a potential new biomarker for clinically localized PCa. However, Zheng et $\mathrm{al}^{26}$ collected serum and urine samples from $100 \mathrm{PCa}$ patients and found no significant difference in the serum and urine GP73 levels between PCa patients before and after treatment, and thus, the ability of GP73 to detect PCa was not obvious. Therefore, the relationship between GP73 and PCa needs further study.

\section{GP73 and breast cancer}

Zhang and $\mathrm{Cao}^{27}$ performed the first study showing that the serum GP73 levels are significantly higher in breast cancer patients than in healthy control individuals, which proves that GP73 is continuously expressed in breast epithelial cells and significantly expressed in invasive breast cancer tissue and serum. Based on this finding, GP73 is hypothesized to be an early indicator of breast cancer. Shui et a ${ }^{28}$ found significantly increased proliferation, decreased apoptosis, and significantly decreased p53 mRNA expression in MCF7 cells overexpressing GP73, which suggests that GP73 might be an upstream regulator of $\mathrm{p} 53$. These data provide new ideas for the diagnosis and gene therapy of breast cancer.

\section{GP73 and pancreatic cancer}

Pancreatic cancer is a highly malignant tumor of the digestive tract that is difficult to diagnose and treat. The early diagnosis rate of pancreatic cancer is not high, and an appropriate marker is urgently needed for its early diagnosis. Duan et a ${ }^{29}$ studied GP73 expression in pancreatic ductal adenocarcinoma (PDAC) tissue and investigated the effect of GP73 on pancreatic cancer cell growth and migration, and their results showed significantly increased GP73 protein expression in PDAC tissues. The growth and motility of PDAC cells are increased upon GP73 overexpression, whereas the downregulation of GP73 expression inhibits cell growth and movement. In addition, the interaction between GP73 and protein kinase B (Akt) increases Akt activity, and GP73 is considered a downstream gene of Ras signaling, which promotes the malignant conversion of normal pancreatic cells. These findings reveal the important role of GP73 in PDAC and suggest that GP73 might be a promising target in the treatment of PDAC in the future.

\section{GP73 and other malignant tumors}

As an important oncogene, GP73 is intimately involved in many types of malignant cancers in addition to those mentioned above, including brain cancer, melanoma, and esophageal cancer. Yuan et $\mathrm{al}^{30}$ found that GP73 mRNA expression is significantly higher in four brain tumor tissues than in normal tissues. Donizy et al ${ }^{13}$ found that high levels of GP73 
in melanoma cells are associated with the characteristics of invasive disease, the disease-free survival (DFS) period, and the cancer-specific overall survival (CSOS) period. Byrne et $\mathrm{al}^{32}$ found that GP73 is secreted by esophageal cancer cells and that the expression and secretion of GP73 promote cell migration and invasion. High GP73 expression has also been detected in seminoma and testicular mesenchymal cell tumors ${ }^{33}$ thus, GP73 can potentially serve as a new marker for evaluating testicular tumors. GP73 mRNA and protein expression in gastric cancer tissues is significantly higher than that in adjacent tissues. A statistical analysis revealed that GP73 expression is closely related to clinical stage, T stage, lymph node metastasis, metastasis, and venous infiltration. Patients with higher GP73 expression have a significantly lower overall survival rate, ${ }^{34}$ which indicates that GP73 has the potential to become a new target in the treatment of gastric cancer.

\section{Conclusion and future perspective}

Multiple studies have shown that GP73 is a new tumor marker that is upregulated in many malignant tumors and is associated with prognosis. GP73 can promote tumor proliferation, apoptosis, invasion, and migration through various mechanisms. However, little is currently known regarding the mechanism of GP73, particularly with respect to chemotherapy resistance and autophagy, and more extensive and in-depth studies and clinical experiments are needed to explore the role of GP73 in the pathogenesis of malignant tumors and to thus develop new strategies for the early diagnosis and treatment of cancer.

\section{Acknowledgments}

This study was supported by the National Natural Science Foundation of People's Republic of China (Grant No 81660498), the Guangxi Natural Science Foundation (Grant No 2016GXNSFBA380090 and 2015GXNSFAA139128), the China Postdoctoral Science Foundation, which is the 60th grant providing funding to the general post-doctoral funding program in the western region (Grant No 2016M602919XB), the Guangxi Medical and Health Appropriate Technology Development and Application Project (Grant No S2017 101), the Basic Ability Enhancement Program for Young and Middle-aged Teachers of Guangxi (Grant No 2017KY0120), and the China Scholarship Council (Grant No 201608455001).

\section{Disclosure}

The authors report no conflicts of interest in this work.

\section{References}

1. Jemal A, Bray F, Center MM, Ferlay J, Ward E, Forman D. Global cancer statistics. CA Cancer J Clin. 2015;61(2):69-90.

2. Kladney RD, Bulla GA, Guo L, et al. GP73, a novel Golgi-localized protein upregulated by viral infection. Gene. 2000;249(1-2):53-65.

3. Bao YX, Yang Y, Zhao HR, et al. [Clinical significance and diagnostic value of Golgi-protein 73 in patients with early-stage primary hepatocellular carcinoma]. Zhonghua Zhong Liu Za Zhi. 2013;35(7):505-508.

4. Ho CC, Luo YH, Chuang TH, Lin P. Quantum dots induced interferon beta expression via TRIF-dependent signaling pathways by promoting endocytosis of TLR4. Toxicology. 2016;344-346:61-70.

5. Ai N, Liu W, Li ZG, Ji H, Li B, Yang G. High expression of GP73 in primary hepatocellular carcinoma and its function in the assessment of transcatheter arterial chemoembolization. Oncol Lett. 2017;14(4): 3953-3958.

6. Pan J, Zhang YF, Yang HY, et al. The response of Golgi protein 73 to transcatheter arterial chemoembolization in patients with hepatocellular carcinoma may relate to the influence of certain chemotherapeutics. Hepatobiliary Pancreat Dis Int. 2015;14(4):406-412.

7. Liu T, Yao M, Liu S, et al. Serum Golgi protein 73 is not a suitable diagnostic marker for hepatocellular carcinoma. Oncotarget. 2017;8(10): 16498-16506

8. Wright LM, Yong S, Picken MM, Rockey D, Fimmel CJ. Decreased survival and hepato-renal pathology in mice with C-terminally truncated GP73 (GOLPH2). Int J Clin Exp Pathol. 2009;2(1):34-47.

9. Ye QH, Zhu WW, Zhang JB, et al. GOLM1 Modulates EGFR/RTK cell-surface recycling to drive hepatocellular carcinoma metastasis. Cancer Cell. 2016;30(3):444-458.

10. Jin D, Tao J, Li D, et al. Golgi protein 73 activation of MMP-13 promotes hepatocellular carcinoma cell invasion. Oncotarget. 2015;6(32): 33523-33533.

11. Zhang S, Ge W, Zou G, et al. MiR-382 targets GOLM1 to inhibit metastasis of hepatocellular carcinoma and its down-regulation predicts a poor survival. Am J Cancer Res. 2018;8(1):120-131.

12. Zhao J, Xu T, Wang F, Cai W, Chen L. miR-493-5p suppresses hepatocellular carcinoma cell proliferation through targeting GP73. Biomed Pharmacother. 2017;90:744-751.

13. Yang Y, Liu Q, Zhang H, et al. Silencing of GP73 inhibits invasion and metastasis via suppression of epithelial-mesenchymal transition in hepatocellular carcinoma. Oncol Rep. 2017;37(2):1182-1188.

14. Liu Y, Zhang X, Sun T, et al. Knockdown of Golgi phosphoprotein 2 inhibits hepatocellular carcinoma cell proliferation and motility. Oncotarget. 2016;7(16):21404-21415.

15. Zhou YY, Jiang JC, You J, Zhou LF. Effect of Golgi phosphoprotein 2 (GOLPH2/GP73) on autophagy in human hepatocellular carcinoma HepG2 cells. Tumour Biol. 2015;36(5):3399-3406.

16. Wang Y, Liu T, Huang P, et al. A novel Golgi protein (GOLPH2)regulated oncolytic adenovirus exhibits potent antitumor efficacy in hepatocellular carcinoma. Oncotarget. 2015;6(15):13564-13578

17. Ye JZ, Yan SM, Yuan CL, et al. GP73 level determines chemotherapeutic resistance in human hepatocellular carcinoma cells. J Cancer. 2018; $9(2): 415-423$

18. Liu X, Fan Z, Zhou C, et al. [The expression and clinical significance of golgi protein GP73 and ki-67 in galloping cancer]. J Prac Med. 2017;33(1):47-50. Chinese.

19. Sun Z, Zeng T. [Expression analysis of serum golgi glycoprotein 73 in lung cancer]. J Guiyang College of Traditional Chinese Medicine. 2013;(3):38-39. Chinese.

20. Han Q, Zhang X, Chen B, et al. [Expression and significance of golgi glycoprotein 73 in NSCLC]. $J$ Med Res. 2016;45(1):44-47. Chinese.

21. Zhang F. [Correlation study of GOLPH2 phosphorylated protein (GOLPH2) with lung cancer and liver cancer]. Univ Sci Technol China. 2016;35(3):44-45. Chinese.

22. Aruna, Li LM. Overexpression of golgi membrane protein 1 promotes non-small-cell carcinoma aggressiveness by regulating the matrix metallopeptidase 13. Am J Cancer Res. 2018;8(3):551-565. 
23. Li W, Zhong W. GOLPH2 expressed in prostate cancer and diagnosis significance. Paper presented at: Guangxi traditional Chinese medicine and traditional Chinese and western medicine men's academic conference. A new development seminar in the national association of Chinese and western medicine. 2015;30(4):23-24.

24. Kristiansen G, Fritzsche FR, Wassermann K, et al. GOLPH2 protein expression as a novel tissue biomarker for prostate cancer: implications for tissue-based diagnostics. Br J Cancer. 2008;99(6):939-948.

25. Laxman B, Morris DS, Yu J, et al. A first-generation multiplex biomarker analysis of urine for the early detection of prostate cancer. Cancer Res. 2008;68(3):645-649.

26. Zheng Y, Lu F, Liu K, Zhou Y, Tao P, Ma C. [GP73 detection value for prostate cancer laboratory diagnosis]. Jiangsu Medical Journal. 2016;(8):968-969. Chinese.

27. Zhang A, Cao B. Generation and characterization of an anti-GP73 monoclonal antibody for immunoblotting and sandwich ELISA. J Biomed Res. 2012;26(6):467-473.

28. Shui Z, Tang X. GP73 regulates the proliferation and apoptosis of McF-7 in breast cancer cells through the p53 signaling pathway. J Third Mil Med Univ. 2018;1:64-69.
29. Duan J, Li X, Huang S, et al. GOLPH2, a gene downstream of ras signaling, promotes the progression of pancreatic ductal adenocarcinoma. Mol Med Rep. 2018;17(3):4187-4194.

30. Yuan Y, Shi L, Wang S. Expression and correlation of CD44 and GP73 in cerebroma tissues. Oncol Lett. 2018;15(4):4958-4962.

31. Donizy P, Kaczorowski M, Biecek P, Halon A, Szkudlarek T, Matkowski R. Golgi-Related Proteins GOLPH2 (GP73/GOLM1) and GOLPH3 (GOPP1/MIDAS) in Cutaneous Melanoma: Patterns of Expression and Prognostic Significance. Int J Mol Sci. 2016;17(10):1619.

32. Byrne AM, Bekiaris S, Duggan G, et al. Golgi phosphoprotein 2 (GOLPH2) is a novel bile acid-responsive modulator of oesophageal cell migration and invasion. Br J Cancer. 2015;113(9):1332-1342.

33. Fritzsche FR, Kristiansen G, Riener MO, Dietel M, Oelrich B. GOLPH2 expression may serve as diagnostic marker in seminomas. BMC Urol. 2010;10:4.

34. Liu G, Zhang Y, He F, et al. Expression of GOLPH2 is associated with the progression of and poor prognosis in gastric cancer. Oncol Rep. 2014; 32(5):2077-2085.
OncoTargets and Therapy

\section{Publish your work in this journal}

OncoTargets and Therapy is an international, peer-reviewed, open access journal focusing on the pathological basis of all cancers, potential targets for therapy and treatment protocols employed to improve the management of cancer patients. The journal also focuses on the impact of management programs and new therapeutic agents and protocols on

\section{Dovepress}

patient perspectives such as quality of life, adherence and satisfaction. The manuscript management system is completely online and includes a very quick and fair peer-review system, which is all easy to use. Visit http://www.dovepress.com/testimonials.php to read real quotes from published authors. 